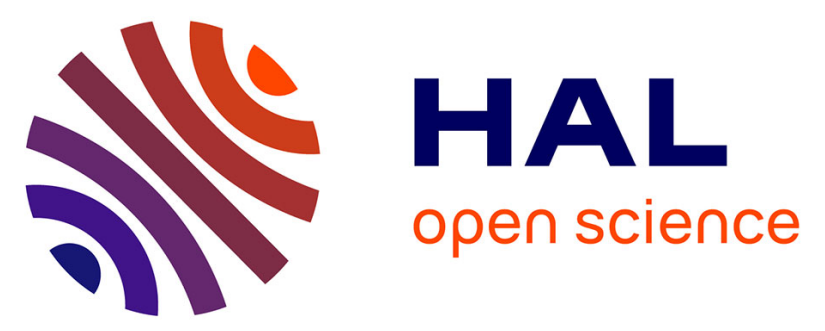

\title{
Promising role of preoperative neutrophil-to-lymphocyte ratio in patients treated with radical nephroureterectomy
}

Mihai Dorin Vartolomei, Romain Mathieu, Vitaly Margulis, Jose A. Karam, Morgan Rouprêt, Ilaria Lucca, Aurélie Mbeutcha, Christian Seitz, Pierre I. Karakiewicz, Harun Fajkovic, et al.

\section{To cite this version:}

Mihai Dorin Vartolomei, Romain Mathieu, Vitaly Margulis, Jose A. Karam, Morgan Rouprêt, et al.. Promising role of preoperative neutrophil-to-lymphocyte ratio in patients treated with radical nephroureterectomy. World Journal of Urology, 2017, 35 (1), pp.121-130. 10.1007/s00345-016-18489. hal-01321614

\section{HAL Id: hal-01321614 \\ https://hal.sorbonne-universite.fr/hal-01321614}

Submitted on 26 May 2016

HAL is a multi-disciplinary open access archive for the deposit and dissemination of scientific research documents, whether they are published or not. The documents may come from teaching and research institutions in France or abroad, or from public or private research centers.
L'archive ouverte pluridisciplinaire HAL, est destinée au dépôt et à la diffusion de documents scientifiques de niveau recherche, publiés ou non, émanant des établissements d'enseignement et de recherche français ou étrangers, des laboratoires publics ou privés.

\section{(c)(1)}

Distributed under a Creative Commons Attribution| 4.0 International License 


\title{
Promising role of preoperative neutrophil-to-lymphocyte ratio in patients treated with radical nephroureterectomy
}

\author{
Mihai Dorin Vartolomei ${ }^{1,2} \cdot$ Romain Mathieu $^{1,3} \cdot$ Vitaly Margulis $^{4} \cdot$ \\ Jose A. Karam ${ }^{5}$ - Morgan Rouprêt ${ }^{6} \cdot$ Ilaria Lucca ${ }^{1,7}$ • Aurélie Mbeutcha ${ }^{1}$. \\ Christian Seitz $^{1} \cdot$ Pierre I. Karakiewicz ${ }^{8} \cdot$ Harun Fajkovic $^{1} \cdot$ Christopher G. Wood $^{5}$. \\ Alon Z. Weizer ${ }^{9}$ Jay D. Raman ${ }^{10}$ - Nathalie Rioux-Leclercq ${ }^{11}$ - Andrea Haitel ${ }^{12}$. \\ Karim Bensalah $^{3} \cdot$ Michael Rink $^{13}$ • Alberto Briganti ${ }^{14}$ - Evanguelos Xylinas ${ }^{15}$. \\ Shahrokh F. Shariat ${ }^{1,4,16,17}$
}

Received: 21 July 2015 / Accepted: 7 May 2016

(C) The Author(s) 2016. This article is published with open access at Springerlink.com

\begin{abstract}
Objective Several retrospective studies with small cohorts reported neutrophil-to-lymphocyte ratio (NLR) as a prognostic marker in upper tract urothelial carcinoma (UTUC) following radical nephroureterectomy (RNU). We aimed at validating the predictive and prognostic role of NLR in a large multi-institutional cohort.

Methods Preoperative NLR was assessed in a multiinstitutional cohort of 2477 patients with UTUC treated with RNU. Altered NLR was defined by a ratio $>2.7$. Logistic regression analyses were performed to assess
\end{abstract}

Mihai Dorin Vartolomei and Romain Mathieu have contributed equally as co-first authors.

Shahrokh F. Shariat

shahrokh.shariat@meduniwien.ac.at

1 Department of Urology, Vienna General Hospital, Medical University of Vienna, Vienna, Austria

2 Department of Cell and Molecular Biology, University of Medicine and Pharmacy, Targu Mures, Romania

3 Department of Urology, Rennes University Hospital, Rennes, France

4 Department of Urology, University of Texas Southwestern Medical Center at Dallas, Dallas, TX, USA

5 Department of Urology, MD Anderson Cancer Center, Houston, TX, USA

6 Academic Department of Urology, La Pitié-Salpetrière Hospital, Assistance Publique-Hôpitaux de Paris, Faculté de Médecine Pierre et Marie Curie, University Paris 6, Paris, France

7 Department of Urology, Centre hospitalier universitaire vaudois, Lausanne, Switzerland

8 Cancer Prognostics and Health Outcomes Unit, University of Montreal Health Centre, Montreal, Canada the association between NLR and lymph node metastasis, muscle-invasive and non-organ-confined disease. The association of altered NLR with recurrence-free survival (RFS) and cancer-specific survival (CSS) was evaluated using Cox proportional hazards regression models.

Results Altered NLR was observed in 1428 (62.8\%) patients and associated with more advanced pathological tumor stage, lymph node metastasis, lymphovascular invasion, tumor necrosis and sessile tumor architecture. In a preoperative model that included age, gender, tumor location and architecture, NLR was an independent predictive factor for the presence of lymph node metastasis, muscle-invasive and non-organ-confined disease

9 Department of Urology, University of Michigan Cancer Center, Ann Arbor, MI, USA

10 Division of Urology, Penn State Milton S. Hershey Medical Center, Hershey, PA, USA

11 Department of Pathology, Rennes University Hospital, Rennes, France

12 Department of Pathology, Medical University of Vienna, Vienna, Austria

13 Department of Urology, University Medical Center Hamburg-Eppendorf, Hamburg, Germany

14 Department of Urology, Vita Salute San Raffaele University, Milan, Italy

15 Department of Urology, Cochin Hospital, Assistance Publique-Hôpitaux de Paris, Paris Descartes University, Paris, France

16 Department of Urology, Weill Cornell Medical College, New York, NY, USA

17 Department of Urology, Comprehensive Cancer Center, Vienna General Hospital, Medical University of Vienna, Währinger Gürtel 18-20, 1090 Vienna, Austria 
$(p<0.001)$. Within a median follow-up of 40 months (IQR 20-76 months), 548 (24.1\%) patients experienced disease recurrence and 453 patients (19.9\%) died from their cancer. Compared to patients with normal NLR, those with altered NLR had worse RFS $(0.003)$ and CSS $(p=0.002)$. In multivariable analyses that adjusted for the effects of standard clinicopathologic features, altered NLR did not retain an independent value. In the subgroup of patients treated with lymphadenectomy in addition to RNU, NLR was independently associated with CSS ( $p=0.03$ ).

Conclusion In UTUC, preoperative NLR is associated with adverse clinicopathologic features and independently predicts features of biologically and clinically aggressive UTUC such as lymph node metastasis, muscle-invasive or non-organ-confined status. NLR may help better risk stratify patients with regard to lymphadenectomy and conservative therapy.

Keywords Neutrophil-to-lymphocyte ratio $\cdot$ Prognostic factor $\cdot$ Urothelium $\cdot$ Carcinoma $\cdot$ Recurrence $\cdot$ Death

\section{Introduction}

Upper tract urothelial carcinoma (UTUC) is a relatively rare disease with an annual incidence of 1-2 cases per 100,000 inhabitants [1]. Despite intense collaborative efforts to improve the knowledge of this disease, its management remains challenging [2]. The preoperative staging is therefore a major concern in UTUC since current imaging has still limited accuracy. Current predictive models based on preoperative parameters propose risk stratification on low-risk and high-risk tumors. These models may guide physicians for treatment decision making regarding the completion of a kidney-sparing procedure or a radical nephroureterectomy (RNU) with or without lymphadenectomy. RNU is indeed the standard treatment for high-risk UTUC. However, up to $30 \%$ of the patients will experience early tumor recurrence after radical treatment and $80 \%$ of these patients will eventually die from their disease $[3,4]$. The prediction of oncologic outcomes is, therefore, another major concern in UTUC. The established prognostic models for UTUC mainly rely on definitive pathological features such as $\mathrm{T}$ stage, architecture, lymphovascular invasion, tumor location and concomitant in situ carcinoma [5]. However, these predictive and prognostic models need further optimization and further studies are needed to identify and validate new preoperative factors. Current efforts are focusing on biological and clinical biomarkers that capture the tumor behavior and reflect its intrinsic aggressiveness.

Markers of inflammatory response have been reported as potential biomarkers of tumor aggressiveness and worse outcome in several malignancies [6]. Indeed, tumor cells interact with their microenvironment and enhance local inflammation by releasing different cytokines and interleukins [7]. Such inflammatory setting could favor tumor progression while modifying levels of routine blood parameters such as C-reactive protein (CRP), leukocytes and derivatives. Several studies reported neutrophil-to-lymphocyte ratio (NLR), that combines neutrophils and lymphocytes, as a predictive factor for the presence of lymph node metastasis or non-organ-confined (NOC) disease in vulva squamous cell carcinoma [8] and in bladder cancer [9], respectively. Other studies demonstrated its prognostic value in pancreatic cancer, colon cancer or breast cancer [10]. In urological neoplasms, its prognostic value has been also reported in bladder cancer [11] and renal cell carcinoma [12]. In UTUC, evidence regarding its prognostic value is, however, limited to recent retrospective studies with relatively small cohorts [13-15], and none is known about its ability to predict adverse pathological features.

The aim of the present study was to externally validate the predictive and prognostic significance of pretreatment NLR in a large multi-institutional cohort from the UTUC collaboration.

\section{Patients and methods}

\section{Patient selection and data collection}

This study obtained an institutional review board approval in each institution, with all participating sites providing institutional data sharing agreements prior to the initiation of the study. 2477 patients treated with RNU for nondistant metastatic UTUC (Ta-T4N0-1M0) between March 1990 and May 2008 at institutions from the international UTUC collaboration were included. Patients with systemic diseases that could interfere with NLR at the time of RNU (such as leukemia, lymphoma, chronic inflammatory diseases, or autoimmune diseases), missing data or a follow-up $<3$ months $(n=203)$ were excluded. No patient received neoadjuvant chemotherapy.

\section{Study variables}

Pretreatment NLR was assessed within the 30 days prior to RNU and defined as altered if ratio $>2.7$. This cutoff was based on previously published results [14]. Demographical, surgical, pathological, NLR (categorically coded according to cutoff) and outcomes data were collected and entered in a computerized database. Histology, tumor stage, grade, location, architecture, presence of lymphovascular invasion (LVI), tumor necrosis and carcinoma in situ (CIS) were confirmed by blinded re-review of the original pathology slides. The 2002 American Joint Committee on 
Cancer-Union International Centre le Cancer (AJCCUICC) Tumor-Node-Metastasis (TNM) classification and the 1998 WHO/International Society of Urologic Pathology (ISUP) consensus classification were used for pathologic staging and grading, respectively.

\section{Management and follow-up}

All patients had a standard RNU with bladder cuff removal with curative intent. A regional lymphadenectomy and adjuvant chemotherapy were completed at the discretion of the urologist. Follow-up was done according to institutional protocols in agreement with local guidelines at the time. Generally, patients were seen postoperatively quarterly for the first year, semiannually in the second year, and annually thereafter. Follow-up visits consisted of a physical examination, serum chemistry evaluation, urinary cytology and endoscopic examination of the bladder. A chest radiography and diagnostic imaging of the contralateral upper urinary tract, with a computerized tomography urogram, an ultrasonography and/or an intravenous pyelography, were performed annually. Chest computerized tomography and bone scan were performed at the discretion of the physicians. Recurrence was defined as any local recurrence (in the retroperitoneum or renal fossa) or distant metastasis. Recurrences in the bladder or contralateral upper urinary tract were considered as second primaries. Outcomes were measured by time to disease recurrence or to cancer-specific death. Cause of death was determined by the treating physician, based on chart review corroborated by death certificates, or by death certificates alone [16].

\section{Statistical analysis}

Associations of NLR with categorical variables were assessed using Chi-square tests, while differences in continuous variables were analyzed using Kruskal-Wallis tests. Kaplan-Meier method was used to estimate recurrence-free survival (RFS) and cancer-specific survival (CSS); log-rank tests were applied for pair-wise comparison of survival. Muscle-invasive disease was defined as $\geq \mathrm{pT} 2$ and/or N+ disease, while NOC as $\geq$ pT3 and/or N+ disease. Logistic regression analysis was performed to assess the association of NLR and other predictive factors with lymph node metastasis, muscle-invasive and NOC disease. Accuracy of the models was calculated using receiver operating characteristic (ROC) analysis. Univariable and multivariable Cox regression models addressed associations of RFS and CSS with potential prognostic factors. Subgroup analyses were done for patients with pTa-2 N0/Nx, pT1-3 N0/Nx, pT3/pT4 N0/Nx disease, high-grade tumors, positive and negative lymph node metastases or, treated with or without lymphadenectomy or adjuvant chemotherapy. All $\mathrm{p}$ values were two-sided, and statistical significance was defined as a $p<0.05$. Statistical analyses were performed using Stata 11.0 statistical software (Stata Corp., College Station, TX, USA).

\section{Results}

\section{Descriptive characteristics and association with altered NLR}

Table 1 summarizes clinicopathologic characteristics of the cohort. A regional lymphadenectomy was performed in 729 patients $(32.1 \%)$. Adjuvant chemotherapy was administered in 217 patients $(9.5 \%)$. Altered NLR was observed in 1428 patients $(62.8 \%)$ and was associated with more advanced pathological tumor stage $(p<0.001)$, LVI $(p<0.001)$, tumor necrosis $(p<0.001)$, sessile architecture $(p<0.001)$ and lymph node metastases $(p<0.001)$ (Table 1).

\section{Association of NLR with high-risk disease and lymph node metastasis}

In univariable analysis, NLR was a predictive factor for lymph node metastasis, muscle-invasive and NOC disease ( $p \leq 0.001$, each) (Table 2). Similarly, kidney location and sessile architecture were independent predictors. In multivariable analysis that adjusted for the effects of age, gender, location and architecture, NLR retained an independent value for the prediction of all three pathologic features ( $p<0.001$, each). Addition of NLR improved by 2 points the accuracies of the models that predicted muscle-invasive and NOC disease (accuracy: 68 and $72 \%$, respectively) and 1 point the one dedicated to the prediction of the lymph node metastatic status (accuracy $=68 \%$ ).

\section{Association of NLR with cancer recurrence and cancer-specific survival}

Within a median follow-up of 40 months (range 20-76 months), 548 patients (24.1\%) experienced disease recurrence and 453 patients (19.9\%) died from their cancer. Patients with altered NLR had worse RFS and CSS than those with normal NLR $(p<0.003$ and $p=0.002$, respectively). 3-year RFS and CSS estimates were: 78 (CI 76.9-82.7 \%) and $85.8 \%$ (CI 83-88.2\%) for patients with normal NLR, and 74.5 (CI 72.0-76.9 \%) and 81.1\% (CI 78.8-83.3\%) for patients with abnormal NLR, respectively (Fig. 1a, b).

Table 3 shows the univariable and multivariable Cox proportional hazard regression analyses in the overall cohort. In univariable analysis, altered NLR was significantly associated with RFS and CSS $(H R=1.30$; 
Table 1 Association of neutrophil-to-lymphocyte ratio (NLR) and clinicopathologic characteristics in 2274 patients treated with radical nephroureterectomy for upper tract urothelial carcinoma

\begin{tabular}{|c|c|c|c|c|}
\hline & All patients & Normal NLR & Altered NLR & $p$ \\
\hline Total, $n(\%)$ & 2274 & $846(37.2)$ & $1428(62.8)$ & \\
\hline Age & & & & 0.66 \\
\hline Median (IQR) & $69(61-76)$ & $69(61-76)$ & $70(62-76)$ & \\
\hline Gender, $n(\%)$ & & & & 0.31 \\
\hline Male & $1527(67.1)$ & $579(68.1)$ & $948(66.4)$ & \\
\hline Female & 747 (32.9) & 267 (31.9) & $480(33.6)$ & \\
\hline Tumor stage, $n(\%)$ & & & & $<0.001$ \\
\hline $\mathrm{pTa}$ & $497(21.8)$ & $199(23.5)$ & $298(20.9)$ & \\
\hline pTis & $48(2.2)$ & $19(2.2)$ & $29(2.3)$ & \\
\hline pT1 & $532(23.4)$ & $244(28.8)$ & $288(20.0)$ & \\
\hline pT2 & $441(19.4)$ & $150(17.8)$ & $291(20.3)$ & \\
\hline pT3 & $671(29.5)$ & $217(25.7)$ & $454(31.8)$ & \\
\hline pT4 & $85(3.7)$ & $17(2.0)$ & $68(4.7)$ & \\
\hline Grade, $n(\%)$ & & & & 0.22 \\
\hline Low & $367(16.14)$ & $147(17.3)$ & $220(15.4)$ & \\
\hline High & $1907(83.86)$ & $699(82.7)$ & $1208(84.6)$ & \\
\hline Lymph node status, $n(\%)$ & & & & 0.001 \\
\hline $\mathrm{pNx}$ & $1545(68.0)$ & $570(67.4)$ & $975(68.2)$ & \\
\hline $\mathrm{pN} 0$ & $545(23.9)$ & $228(27.0)$ & $317(22.2)$ & \\
\hline $\mathrm{pN} 1$ & $184(8.1)$ & $48(5.6)$ & $136(9.6)$ & \\
\hline Lymphovascular invasion, $n(\%)$ & & & & $<0.001$ \\
\hline Yes & 499 (21.9) & $143(16.9)$ & $356(24.9)$ & \\
\hline No & $1775(78.1)$ & $703(83.1)$ & $1072(75.1)$ & \\
\hline Concomitant carcinoma in situ, $n(\%)$ & & & & 0.17 \\
\hline Yes & $528(23.2)$ & $183(21.6)$ & $345(24.1)$ & \\
\hline No & $1746(76.8)$ & $663(78.4)$ & $1083(75.9)$ & \\
\hline Multifocality, $n(\%)$ & & & & 0.16 \\
\hline Yes & $538(23.7)$ & $214(25.3)$ & $324(22.7)$ & \\
\hline No & $1736(76.3)$ & $632(74.7)$ & $1104(77.3)$ & \\
\hline Necrosis, $n(\%)$ & & & & $<0.001$ \\
\hline Yes & $516(22.7)$ & $129(15.2)$ & $387(27.1)$ & \\
\hline No & $1758(77.3)$ & $717(84.8)$ & $1041(72.9)$ & \\
\hline Architecture, $n(\%)$ & & & & 0.001 \\
\hline Papillary & $1751(77.0)$ & $684(80.9)$ & $1067(74.8)$ & \\
\hline Sessile & $523(23.0)$ & $162(19.2)$ & $361(25.2)$ & \\
\hline Location & & & & 0.67 \\
\hline Kidney & $1448(63.7)$ & $534(63.1)$ & $914(64.0)$ & \\
\hline Ureter & $826(36.3)$ & $312(36.9)$ & $514(36.0)$ & \\
\hline
\end{tabular}

Statistically significant results are shown in bold $p=0.003$ and HR $=1.36 ; p=0.002$, respectively); however, when adjusted for the effects of clinicopathologic features, NLR did not retain its statistical significance for both endpoints ( $\mathrm{HR}=1.05 ; p=0.59$ and $\mathrm{HR}=1.07 ; p=0.48$, respectively). Similar results were observed in all subgroup analyses performed according to $\mathrm{T}$ stage, grade, lymph node status or adjuvant chemotherapy (Table 4).

\section{Association of NLR with cancer recurrence and cancer-specific survival after RNU with lymphadenectomy}

The median number of lymph nodes removed during lymphadenectomy was 5 (IQR 2-10). Among the patients treated with lymphadenectomy, 239 (32.8\%) experienced 


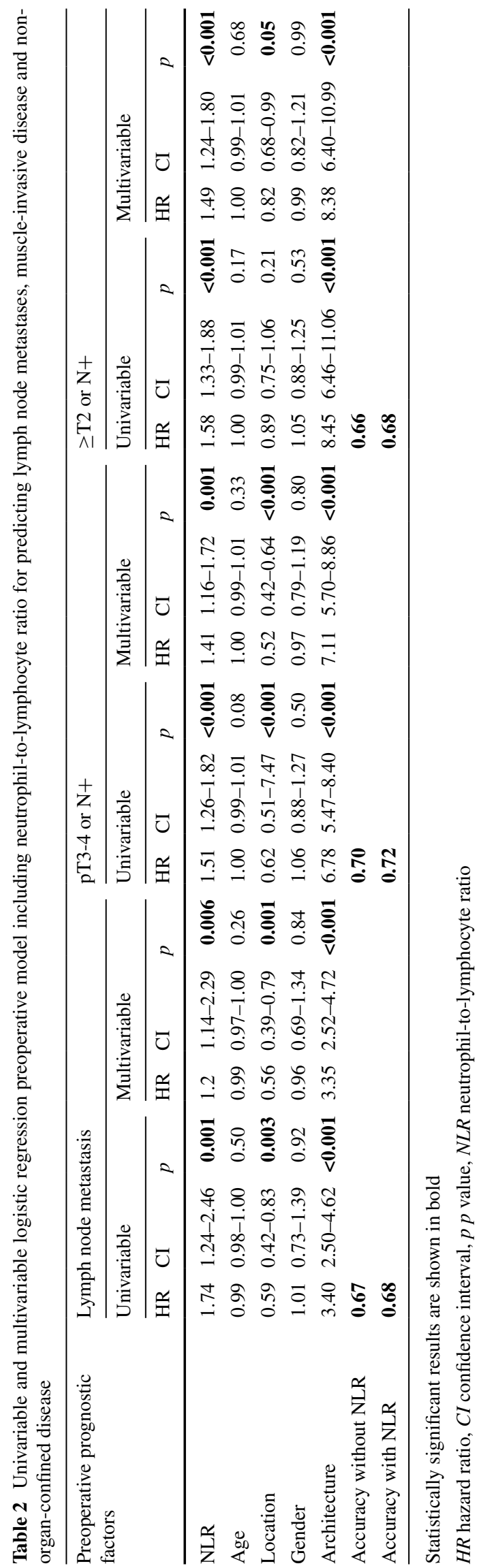

tumor recurrence and $192(26.3 \%)$ died from the disease. NLR was altered in $170(71.1 \%)$ and $141(73.4 \%)$ patients, respectively. On Kaplan-Mayer analysis, altered NLR was associated with both worse RFS and CSS ( $p<0.001$, each). 3-year RFS and CSS were 62.1 and $72.9 \%$ in patients with altered NLR, compared to 73.9 and $84 \%$ in patients with normal NLR (Fig. 1c, d). NLR was associated with RFS (HR 1.63; IC 1.23-2.16, $p<0.001$ ) and CSS (HR 1.79; IC $1.30-2.47, p<0.001$ ) on univariable analyses. When adjusted for the effects of clinicopathologic features, NLR retained its statistical significant association with CSS (HR 1.43; IC 1.02-2.00, $p=0.03$ ), but not with RFS (HR 1.29; IC $0.96-1.73, p=0.08)$ (Table 5).

\section{Discussion}

In the present study, we assessed the significance of preoperative NLR in a large multi-institutional cohort of patients with UTUC treated by RNU. We demonstrated that abnormal NLR was not only associated with adverse pathological features and worse oncologic outcomes, but also predicted the presence of lymph node metastases, muscle-invasive and NOC disease. Therefore, the potential of NLR could be in the preoperative clinical decision making regarding lymphadenectomy indication and extent, and patient counseling regarding conservative therapy.

Using a ratio of 2.7, we observed that almost two-thirds of the patients had an altered preoperative NLR. Altered NLR was associated with features of biologically and clinically aggressive UTUC such as advanced tumor stage, LVI, tumor necrosis, sessile architecture and lymph node metastases. These findings confirm the results reported in smaller cohorts [13-15]. The potential reasons for such associations remain hypothetical. NLR could reflect a balance between neutrophilia and lymphopenia that results from the relationship between the immune system and tumorigenesis. On the one hand, the secretion of granulocyte colonystimulating factor (G-CSF) by tumor cells may promote neutrophils production in bone marrow and recruitment of the neutrophils in the tumor environment. De Larco et al. [7] showed that these "tumor-associated neutrophils" could have a role in the tumor microenvironment and local angiogenesis. Neoangiogenesis could therefore promote tumor progression and migration of tumor cells. On the other hand, lymphopenia may be responsible for poor immune response against tumor and favor tumor aggressiveness and progression [17]. Even if this association is of great interest, it does not ensure the independent value of NLR to predict adverse pathological features at final pathology.

We demonstrated, therefore, the ability of NLR to independently predict lymph node metastasis, muscle-invasive and NOC disease in a preoperative model that included 

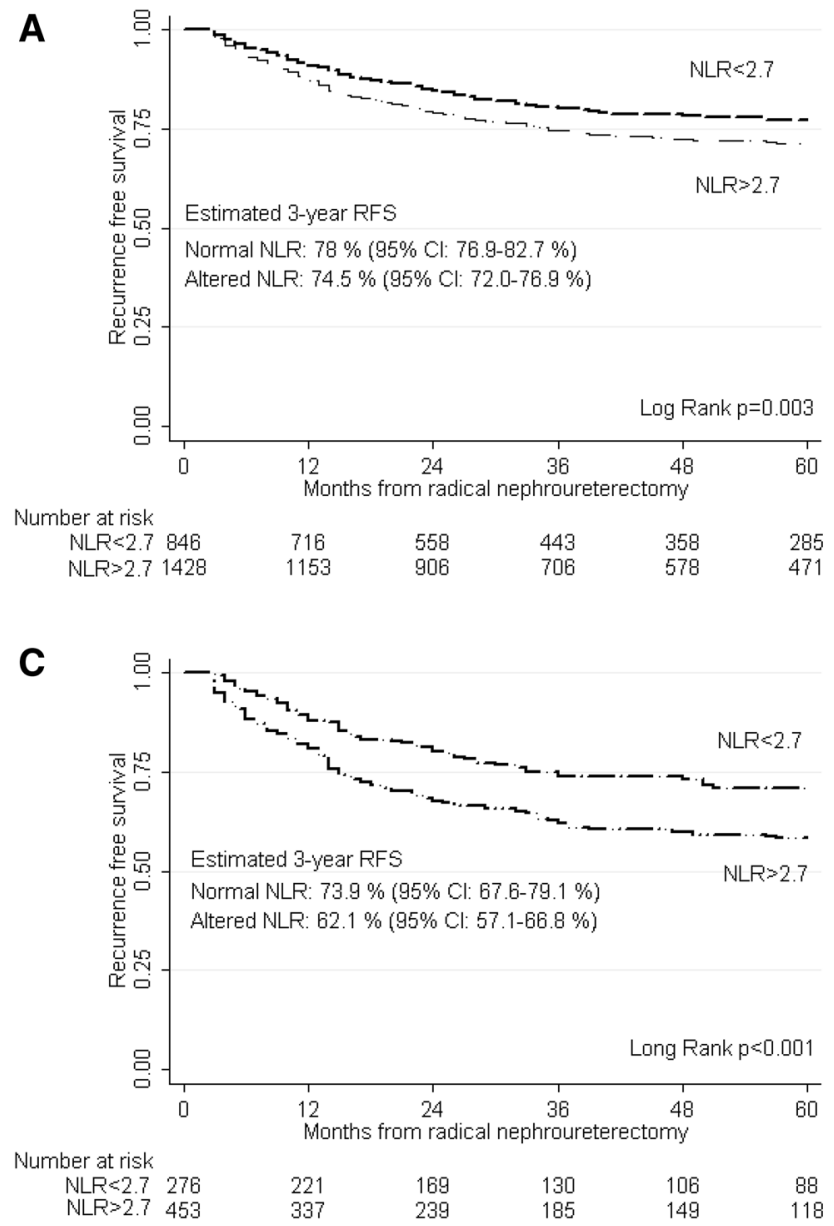

Fig. 1 Kaplan-Meier estimates of recurrence-free survival and cancer-specific survival stratified by NLR status in 2274 patients with UTUC treated with RNU alone (a, b, respectively), and 729 patients with UTUC treated with RNU and lymphadenectomy (c, d, respec-

patient age, gender, tumor location and architecture. This preoperative model may help physicians identify patients who should be proposed a radical treatment with RNU or a lymphadenectomy during RNU [18]. Previous models have been proposed to identify such patients and adapt clinical decision making [19-21]. Margulis et al. [19] developed a model to predict muscle-invasive disease using preoperative clinicopathologic features such as age, gender, tumor location, architecture and grade at biopsy. Another predictive model combined high grade, tumor location, local invasion and hydronephrosis on imaging and achieved 71 and $70 \%$ accuracies for predicting muscle-invasive and NOC disease, respectively [20]. We constructed a model including NLR but did not consider, however, grade at biopsy and data from the preoperative imaging. Our model only relies on easy accessible factors and demonstrates accuracies of 68-72.5\% for the prediction of NOC, muscle-invasive or positive lymph node disease. Further improvement of these models may be obtained, however, by the combination of
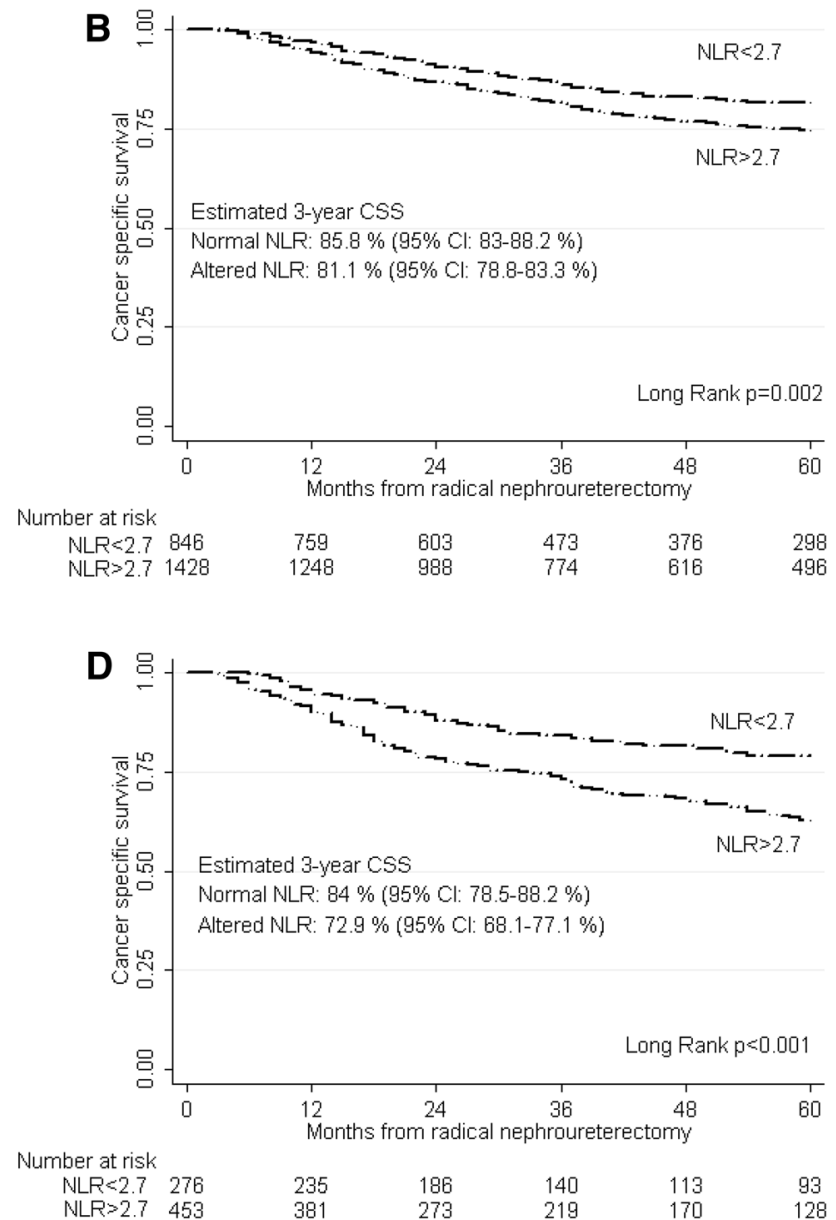

tively). RFS recurrence-free survival, $C S S$ cancer-specific survival, $C I$ confidence interval, NLR neutrophil-to-lymphocyte ratio, UTUC upper tract urothelial carcinoma, $R N U$ radical nephroureterectomy

NLR with other routinely available biomarkers as CRP [22].

We demonstrated NLR was associated with worse oncologic outcomes such as RFS and CSS. However, adjusted for standard pathologic prognostic factors in UTUC, NLR did not retain statistical significance. Most of the previous studies reported, however, independent predictive value for RFS [15], CSS [13, 14] and OS [14]. The discrepancies between these studies and our findings could be explained by several reasons. First, initial studies defined the optimal cutoff for NLR as 2.7 [14] or 3 [13] based on the interpretations of iterative Cox or ROC analysis observed in the cohorts. Only one study externally validated the prognostic value of NLR using a predefined threshold of 3 [15]. To our knowledge, we are the first to propose an external validation with the 2.7 cutoff proposed by Dalpiaz et al. [14]. Second, the parameters used in multivariable analysis proposed in these studies varied widely. For example, Tanaka et al. only adjusted on age, N status, stage and LVI. 
Table 3 Univariable and multivariable Cox regression analyses predicting recurrence and cancer-specific mortality of 2274 patients treated with radical nephroureterectomy for upper tract urothelial carcinoma

\begin{tabular}{|c|c|c|c|c|c|c|c|c|c|c|c|c|}
\hline & \multicolumn{6}{|c|}{ Recurrence-free survival } & \multicolumn{6}{|c|}{ Cancer-specific survival } \\
\hline & \multicolumn{3}{|c|}{ Univariable } & \multicolumn{3}{|c|}{ Multivariable } & \multicolumn{3}{|c|}{ Univariable } & \multicolumn{3}{|c|}{ Multivariable } \\
\hline & HR & $95 \% \mathrm{CI}$ & $p$ value & HR & $95 \% \mathrm{CI}$ & $p$ value & HR & $95 \% \mathrm{CI}$ & $p$ value & HR & $95 \% \mathrm{CI}$ & $p$ value \\
\hline Age & 1.01 & $1.00-1.02$ & $<0.001$ & 1.01 & $1.00-1.02$ & 0.001 & 1.02 & $1.01-1.03$ & $<0.001$ & 1.02 & $1.01-1.03$ & $<0.001$ \\
\hline Gender & 1.10 & $0.92-1.31$ & 0.27 & 1.01 & $0.84-1.21$ & 0.89 & 1.06 & $0.87-1.29$ & 0.52 & 0.94 & $0.77-1.15$ & 0.58 \\
\hline \multicolumn{13}{|l|}{ Stage } \\
\hline $\mathrm{Ta}$ & Ref. & & & & & & & & & & & \\
\hline Tis & 2.98 & $1.30-6.83$ & 0.01 & 1.61 & $0.94-7.25$ & 0.066 & 3.58 & $1.44-8.88$ & 0.006 & 3.37 & $1.08-10.56$ & 0.03 \\
\hline $\mathrm{T} 1$ & 2.16 & $1.38-3.37$ & $<0.001$ & 1.87 & $0.89-3.91$ & 0.095 & 2.00 & $1.18-3.37$ & 0.009 & 1.66 & $0.70-3.92$ & 0.24 \\
\hline $\mathrm{T} 2$ & 5.01 & $3.30-7.59$ & $<0.001$ & 3.84 & $1.86-7.92$ & $<0.001$ & 5.54 & $3.45-8.91$ & $<0.001$ & 4.11 & $1.79-9.45$ & $<0.001$ \\
\hline T3 & 10.29 & $6.97-15.19$ & $<0.001$ & 6.96 & $3.40-14.21$ & $<0.001$ & 11.93 & $7.63-18.65$ & $<0.001$ & 7.85 & $3.45-17.88$ & $<0.001$ \\
\hline $\mathrm{T} 4$ & 39.75 & $25.36-62.29$ & $<0.001$ & 19.94 & $9.26-42.96$ & $<0.001$ & 46.14 & 27.74-76.75 & $<0.001$ & 21.30 & $8.86-51.22$ & $<0.001$ \\
\hline Grade & 6.17 & $3.94-9.64$ & $<0.001$ & 1.10 & $0.48-2.50$ & 0.81 & 6.93 & $4.14-11.60$ & $<0.001$ & 1.17 & $0.45-3.03$ & 0.73 \\
\hline $\begin{array}{l}\text { Lymphovascu- } \\
\text { lar invasion }\end{array}$ & 3.13 & $2.64-3.71$ & $<0.001$ & 1.24 & $1.02-1.52$ & 0.029 & 3.39 & $2.81-4.09$ & $<0.001$ & 1.35 & $1.08-1.67$ & 0.006 \\
\hline Architecture & 3.30 & $2.78-3.91$ & $<0.001$ & 1.37 & $1.12-1.66$ & 0.002 & 3.46 & $2.87-4.17$ & $<0.001$ & 1.36 & $1.10-1.69$ & 0.004 \\
\hline $\begin{array}{l}\text { Carcinoma } \\
\text { in situ }\end{array}$ & 1.67 & $1.39-2.00$ & $<0.001$ & 1.23 & $1.01-1.50$ & 0.035 & 1.57 & $1.28-1.92$ & $<0.001$ & 1.08 & $0.87-1.35$ & 0.46 \\
\hline Necrosis & 2.22 & $1.86-2.64$ & $<0.001$ & 0.98 & $0.81-1.19$ & 0.90 & 2.25 & $1.86-2.74$ & $<0.001$ & 1.00 & $0.81-1.23$ & 0.98 \\
\hline Multifocality & 1.31 & $1.08-1.58$ & 0.004 & 0.91 & $0.74-1.11$ & 0.36 & 1.33 & $1.08-1.64$ & 0.006 & 0.95 & $0.76-1.70$ & 0.66 \\
\hline Location & 1.11 & $0.94-1.32$ & 0.19 & 1.31 & $1.09-1.57$ & 0.003 & 1.16 & $0.96-1.40$ & 0.11 & 1.39 & $1.14-1.70$ & $<0.001$ \\
\hline $\begin{array}{l}\text { Lymph node } \\
\text { metastases } \\
\mathrm{Nx}\end{array}$ & Ref. & & & & & & & & & & & \\
\hline No & 1.13 & $0.92-1.40$ & 0.22 & 0.91 & $0.73-1.12$ & 0.39 & 0.99 & $0.77-1.26$ & 0.94 & 0.77 & $0.60-0.98$ & 0.04 \\
\hline N1 & 5.26 & $4.25-6.51$ & $<0.001$ & 2.10 & $1.64-2.68$ & $<0.001$ & 5.24 & $4.16-6.59$ & $<0.001$ & 1.96 & $1.51-2.56$ & $<0.001$ \\
\hline NLR & 1.30 & $1.09-1.56$ & 0.003 & 1.05 & $0.87-1.26$ & 0.59 & 1.36 & $1.11-1.66$ & 0.002 & 1.07 & $0.87-1.31$ & 0.48 \\
\hline
\end{tabular}

$C I$ confidence interval, $H R$ hazard ratio, $N L R$ neutrophil-to-lymphocyte ratio

Table 4 Univariable and multivariable Cox regression analyses for prediction of recurrence and cancer-specific mortality according NLR status in subgroups of patients treated with radical nephroureterectomy

\begin{tabular}{|c|c|c|c|c|c|c|c|c|c|c|c|c|}
\hline \multirow[t]{3}{*}{ Patient subgroups } & \multicolumn{6}{|c|}{ Recurrence-free survival } & \multicolumn{6}{|c|}{ Cancer-specific survival } \\
\hline & \multicolumn{3}{|c|}{ Univariable } & \multicolumn{3}{|c|}{ Multivariable } & \multicolumn{3}{|c|}{ Univariable } & \multicolumn{3}{|c|}{ Multivariable } \\
\hline & HR & $95 \% \mathrm{CI}$ & $p$ value & HR & $95 \% \mathrm{CI}$ & $p$ value & HR & $95 \% \mathrm{CI}$ & $p$ value & HR & $95 \% \mathrm{CI}$ & $p$ value \\
\hline pTa-4 high grade & 1.28 & $1.07-1.54$ & 0.007 & 1.05 & $0.87-1.26$ & 0.65 & 1.34 & $1.09-1.64$ & 0.005 & 1.06 & $0.87-1.31$ & 0.54 \\
\hline pTa-2 pN0/Nx & 0.98 & $0.74-1.31$ & 0.91 & 0.85 & $0.63-1.13$ & 0.27 & 1.02 & $0.73-1.41$ & 0.92 & 0.86 & $0.62-1.20$ & 0.38 \\
\hline pT1-3 pN0/Nx & 1.07 & $0.87-1.33$ & 0.51 & 0.92 & $0.74-1.14$ & 0.43 & 1.20 & $0.94-1.52$ & 0.14 & 1.01 & $0.79-1.29$ & 0.92 \\
\hline pT3/pT4 N0/Nx & 1.16 & $0.87-1.53$ & 0.31 & 1.12 & $0.84-1.49$ & 0.44 & 1.26 & $0.92-1.72$ & 0.1 & 1.20 & $0.87-1.64$ & 0.27 \\
\hline $\mathrm{pT} 2-4 \mathrm{pN} 0 / \mathrm{Nx}$ & 1.08 & $0.86-1.36$ & 0.51 & 1.01 & $0.80-1.28$ & 0.90 & 1.11 & $0.86-1.43$ & 0.40 & 1.03 & $0.80-1.33$ & 0.82 \\
\hline pTa-4 pN0 & 1.30 & $0.90-1.89$ & 0.16 & 1.17 & $0.79-1.74$ & 0.42 & 1.69 & $1.07-2.67$ & 0.02 & 1.47 & $0.91-2.38$ & 0.1 \\
\hline pTa-4 pN1 & 1.54 & $0.99-2.39$ & 0.05 & 1.30 & $0.81-2.09$ & 0.27 & 1.39 & $0.88-2.19$ & 0.15 & 1.20 & $0.73-1.98$ & 0.46 \\
\hline
\end{tabular}

$C I$ confidence interval, $H R$ hazard ratio

We performed a multivariable analysis using most of the clinicopathologic parameters published in the literature $[3,23,24]$. When we limited our multivariable analysis to the parameters used in established prognostic models [3,
23-25], NLR did not demonstrate independent prognostic value, however (data not shown). Third, populations were different regarding the clinicopathologic characteristics. Luo et al. [13] only included patients with $\mathrm{pT}$ stage $<4$ and 
Table 5 Univariable and multivariable Cox regression analyses predicting the recurrence and cancer-specific mortality of 729 patients treated with radical nephroureterectomy and lymphadenectomy for upper tract urothelial carcinoma

\begin{tabular}{|c|c|c|c|c|c|c|c|c|c|c|c|c|}
\hline & \multicolumn{6}{|c|}{ Recurrence-free survival } & \multicolumn{6}{|c|}{ Cancer-specific survival } \\
\hline & \multicolumn{3}{|c|}{ Univariable } & \multicolumn{3}{|c|}{ Multivariable } & \multicolumn{3}{|c|}{ Univariable } & \multicolumn{3}{|c|}{ Multivariable } \\
\hline & HR & $95 \% \mathrm{CI}$ & $p$ value & HR & $95 \% \mathrm{CI}$ & $p$ value & HR & $95 \% \mathrm{CI}$ & $p$ value & HR & $95 \% \mathrm{CI}$ & $p$ value \\
\hline Age & 1.01 & $1.00-1.02$ & 0.01 & 1.01 & $0.99-1.02$ & 0.06 & 1.02 & $1.00-1.03$ & $<0.001$ & 1.02 & $1.00-1.03$ & 0.005 \\
\hline Gender & 1.23 & $0.95-1.60$ & 0.11 & 1.14 & $0.86-1.49$ & 0.34 & 1.06 & $0.79-1.43$ & 0.66 & 0.93 & $0.68-1.27$ & 0.67 \\
\hline \multicolumn{13}{|l|}{ Stage } \\
\hline $\mathrm{Ta}$ & Ref. & & & & & & & & & & & \\
\hline Tis & 1.06 & $0.12-8.87$ & 0.95 & 1.47 & $0.13-16.43$ & 0.75 & 1.52 & $0.17-13.06$ & 0.70 & 3.81 & $0.23-61.67$ & 0.34 \\
\hline $\mathrm{T} 1$ & 2.34 & $0.93-5.90$ & 0.07 & 2.76 & $0.63-11.98$ & 0.17 & 1.41 & $0.47-4.23$ & 0.53 & 2.63 & $0.33-20.90$ & 0.36 \\
\hline $\mathrm{T} 2$ & 5.24 & $2.23-12.31$ & $<0.001$ & 5.37 & $1.28-22.42$ & 0.02 & 4.34 & $1.69-11.13$ & 0.002 & 6.64 & $0.89-49.14$ & 0.06 \\
\hline $\mathrm{T} 3$ & 10.22 & $4.50-23.17$ & $<0.001$ & 8.68 & $2.12-35.50$ & 0.003 & 10.03 & $4.09-24.61$ & $<0.001$ & 12.34 & $1.70-84.46$ & 0.01 \\
\hline $\mathrm{T} 4$ & 32.13 & $13.57-76.07$ & $<0.001$ & 19.95 & $4.66-85.29$ & $<0.001$ & 33.0 & $12.90-84.60$ & $<0.001$ & 25.92 & $3.45-194.75$ & 0.002 \\
\hline Grade & 6.76 & $2.51-18.18$ & $<0.001$ & 0.73 & $0.13-4.04$ & 0.72 & 5.30 & $1.97-14.29$ & $<0.001$ & 0.36 & $0.04-3.26$ & 0.36 \\
\hline $\begin{array}{l}\text { Lymphovascular } \\
\text { invasion }\end{array}$ & 2.45 & $1.90-3.16$ & $<0.001$ & 0.88 & $0.66-1.18$ & 0.42 & 2.92 & $2.19-3.88$ & $<0.001$ & 1.05 & $0.76-1.45$ & 0.74 \\
\hline Architecture & 3.05 & $2.36-3.94$ & $<0.001$ & 1.48 & $1.10-2.00$ & 0.009 & 3.39 & $2.55-4.52$ & $<0.001$ & 1.58 & $1.13-2.20$ & 0.007 \\
\hline Carcinoma in situ & 1.38 & $1.06-1.79$ & 0.01 & 1.17 & $0.88-1.56$ & 0.26 & 1.31 & $0.97-1.77$ & 0.07 & 0.94 & $0.68-1.31$ & 0.74 \\
\hline Necrosis & 1.97 & $1.52-2.54$ & $<0.001$ & 1.02 & $0.77-1.35$ & 0.85 & 1.97 & $1.48-2.62$ & $<0.001$ & 1.02 & $0.75-1.39$ & 0.86 \\
\hline Multifocality & 1.12 & $0.84-1.48$ & 0.42 & 0.82 & $0.60-1.11$ & 0.20 & 1.32 & $0.97-1.80$ & 0.07 & 0.99 & $0.71-1.39$ & 0.98 \\
\hline Location & 0.97 & $0.74-1.27$ & 0.87 & 1.23 & $0.93-1.64$ & 0.13 & 1.02 & $0.76-1.37$ & 0.87 & 1.31 & $0.95-1.79$ & 0.09 \\
\hline Lymph node metastases & 4.61 & $3.57-5.96$ & $<0.001$ & 2.62 & $1.95-3.52$ & $<0.001$ & 5.23 & $3.93-6.97$ & $<0.001$ & 2.75 & $1.98-3.83$ & $<0.001$ \\
\hline NLR & 1.63 & $1.23-2.16$ & $<0.001$ & 1.29 & $0.96-1.73$ & 0.08 & 1.79 & $1.30-2.47$ & $<0.001$ & 1.43 & $1.02-2.00$ & 0.03 \\
\hline
\end{tabular}

Statistically significant results are shown in bold

$C I$ confidence interval, $H R$ hazard ratio, $N L R$ neutrophil-to-lymphocyte ratio

without lymph node metastases. Conversely, half cohort of Tanaka et al. [15] had locally advanced disease. However, none of the subgroup analyses we performed with advanced disease or localized disease demonstrated independent prognostic value for NLR.

We demonstrated, however, that NLR would be an independent prognostic factor regarding CSS in patients who were treated with lymphadenectomy. In previous studies, status of lymphadenectomy was not routinely performed [13-15]. Staging benefit of lymphadenectomy in UTUC is noteworthy [26]. Xylinas et al. [27] showed, therefore, that all patients treated with RNU for UTUC should receive LND to ensure accurate nodal staging. In the future, metastatic patients may also receive immune checkpoint inhibitors such as anti-PDL1. Preliminary results in bladder cancer suggest that circulating inflammatory markers may predict response to immune checkpoint inhibitors [28]. Therefore, NLR could be of great value in the identification of patients with metastatic status that may benefit from immune checkpoint inhibitors.

Although this is the largest study that investigated the predictive and prognostic value of pretreatment NLR with outcomes after RNU, our study has some limitations that should be considered. First, its retrospective and multicentric status may be responsible for variations in laboratory, pathological and surgical workup that could confound the results. In the present study, NLR was determined preoperatively with a predefined cutoff and analyzed as a categorical variable. We did not complete these investigations with exact levels of NLR as neutrophils and lymphocytes counts were measured with different assays. Continuously coded NLR or different thresholds may have provided different conclusions. Assessment of NLR at different points in time may also have excluded confounders, such as occult infectious diseases without any symptoms preoperative, autoimmune diseases responsible for temporally changes. Finally, we only investigated NLR. A growing evidence suggests that derivatives of NLR or its combination with other preoperative markers of systemic inflammation may be helpful in the prediction of oncologic outcomes in UTUC and warrants further investigations [29-31].

\section{Conclusion}

In UTUC, NLR is associated with adverse clinicopathologic features and worse oncologic outcomes. However, 
its prognostic role may be limited to patients treated with lymphadenectomy. Conversely, all patients could benefit in the preoperative setting from its ability to independently predict lymph node metastasis, muscle-invasive and NOC disease. NLR may be therefore a useful biomarker in clinical decision making regarding a radical treatment and the completion and extent of a lymphadenectomy.

Acknowledgments Open access funding provided by Medical University of Vienna. R. M. is supported by the Scholarship Foundation of the Republic of Austria-OeAD and by the EUSP Scholarship-European Association of Urology. M. D. V is supported by the Sectoral Operational Programme Human Resources Development (SOP HRD), financed from the European Social Fund and by the Romanian Government under the contract number POSDRU/159/1.5/S/133377 and University of Medicine and Pharmacy, Targu Mures Romania contract number 17800/2015.

Authors' contribution Mihai Dorin Vartolomei, Romain Mathieu, Vitaly Margulis, Shahrokh F. Shariat were involved in protocol/project development; Mihai Dorin Vartolomei, Romain Mathieu, Ilaria Lucca, Aurelie Mbeutcha, Tobias Klatte, Vitaly Margulis, Jose A.Karam, Morgan Rouprêt, Christian Seitz, Pierre I. Karakiewicz, Harun Fajkovic, Christopher G. Wood, Alon Z. Weizer, Jay D. Raman, Nathalie RiouxLeclercq, Andrea Haitel, Karim Bensalah, Michael Rink, Albetro Briganti, Shahrokh F. Shariat collected or managed data; Mihai Dorin Vartolomei, Romain Mathieu, Tobias Klatte, Vitaly Margulis, Jose A.Karam, Morgan Rouprêt, Christian Seitz, Pierre I. Karakiewicz, Harun Fajkovic, Christopher G. Wood, Alon Z. Weizer, Jay D. Raman, Nathalie RiouxLeclercq, Andrea Haitel, Karim Bensalah, Michael Rink, Alberto Briganti, Evanguelos Xylinas, Shahrokh F. Shariat analyzed data; Mihai Dorin Vartolomei, Romain Mathieu, Ilaria Lucca, Aurelie Mbeutcha, Tobias Klatte, Vitaly Margulis, Jose A.Karam, Morgan Rouprêt, Christian Seitz, Pierre I. Karakiewicz, Harun Fajkovic, Christopher G. Wood, Alon Z. Weizer, Jay D. Raman, Nathalie Rioux-Leclercq, Andrea Haitel, Karim Bensalah, Michael Rink, Alberto Briganti, Evanguelos Xylinas, Shahrokh F. Shariat were involved in manuscript writing/editing.

\section{Compliance with ethical standards}

Conflict of interest The authors declare that they have no conflict of interest.

Ethical standards This study has been approved by the appropriate ethics committee.

Open Access This article is distributed under the terms of the Creative Commons Attribution 4.0 International License (http://creativecommons.org/licenses/by/4.0/), which permits unrestricted use, distribution, and reproduction in any medium, provided you give appropriate credit to the original author(s) and the source, provide a link to the Creative Commons license, and indicate if changes were made.

\section{References}

1. Siegel RL, Miller KD, Jemal A (2015) Cancer statistics, 2015. CA Cancer J Clin 65(1):5-29

2. Mathieu R, Bensalah K, Lucca I, Mbeutcha A, Rouprêt M, Shariat SF (2015) Upper urinary tract disease: what we know today and unmet needs. Transl Androl Urol 4(3):261-272
3. Cha EK, Shariat SF, Kormaksson M, Novara G, Chromecki TF, Scherr DS et al (2012) Predicting clinical outcomes after radical nephroureterectomy for upper tract urothelial carcinoma. Eur Urol 61(4):818-825

4. Rink M, Sjoberg D, Comploj E, Margulis V, Xylinas E, Lee RK et al (2012) Risk of cancer-specific mortality following recurrence after radical nephroureterectomy. Ann Surg Oncol 19(13):4337-4344

5. Xylinas E, Kluth L, Mangal S, Roupret M, Karakiewicz PI, Shariat SF (2013) Predictive tools for clinical decision-making and counseling of patients with upper tract urothelial carcinoma. World J Urol 31(1):31-36

6. Roxburgh CSD, McMillan DC (2010) Role of systemic inflammatory response in predicting survival in patients with primary operable cancer. Fut Oncol Lond Engl 6(1):149-163

7. De Larco JE, Wuertz BRK, Furcht LT (2004) The potential role of neutrophils in promoting the metastatic phenotype of tumors releasing interleukin-8. Clin Cancer Res Off J Am Assoc Cancer Res 10(15):4895-4900

8. Ertas IE, Gungorduk K, Akman L, Ozdemir A, Terek MC, Ozsaran A et al (2013) Can preoperative neutrophil: lymphocyte and platelet: lymphocyte ratios be used as predictive markers for lymph node metastasis in squamous cell carcinoma of the vulva? Eur J Obstet Gynecol Reprod Biol 171(1):138-142

9. Potretzke A, Hillman L, Wong K, Shi F, Brower R, Mai S et al (2014) NLR is predictive of upstaging at the time of radical cystectomy for patients with urothelial carcinoma of the bladder. Urol Oncol 32(5):631-636

10. Templeton AJ, McNamara MG, Šeruga B, Vera-Badillo FE, Aneja P, Ocaña A et al (2014) Prognostic role of neutrophil-tolymphocyte ratio in solid tumors: a systematic review and metaanalysis. J Natl Cancer Inst 106(6):dju124

11. Lucca I, Jichlinski P, Shariat SF, Rouprêt M, Rieken M, Kluth LA et al (2015) The neutrophil-to-lymphocyte ratio as a prognostic factor for patients with urothelial carcinoma of the bladder following radical cystectomy: validation and meta-analysis. Eur Urol Focus [Internet]. http://www.sciencedirect.com/science/ article/pii/S2405456915000115

12. de Martino M, Pantuck AJ, Hofbauer S, Waldert M, Shariat SF, Belldegrun AS et al (2013) Prognostic impact of preoperative neutrophil-to-lymphocyte ratio in localized nonclear cell renal cell carcinoma. J Urol 190(6):1999-2004

13. Luo H-L, Chen Y-T, Chuang Y-C, Cheng Y-T, Lee W-C, Kang C-H et al (2014) Subclassification of upper urinary tract urothelial carcinoma by the neutrophil-to-lymphocyte ratio (NLR) improves prediction of oncological outcome. BJU Int 113(5b):E144-E149

14. Dalpiaz O, Ehrlich GC, Mannweiler S, Hernández JMM, Gerger A, Stojakovic T et al (2014) Validation of pretreatment neutrophil-lymphocyte ratio as a prognostic factor in a European cohort of patients with upper tract urothelial carcinoma. BJU Int 114(3):334-339

15. Tanaka N, Kikuchi E, Kanao K, Matsumoto K, Shirotake S, Miyazaki Y et al (2014) A multi-institutional validation of the prognostic value of the neutrophil-to-lymphocyte ratio for upper tract urothelial carcinoma treated with radical nephroureterectomy. Ann Surg Oncol 21(12):4041-4048

16. Rink M, Fajkovic H, Cha EK, Gupta A, Karakiewicz PI, Chun FK et al (2012) Death certificates are valid for the determination of cause of death in patients with upper and lower tract urothelial carcinoma. Eur Urol 61(4):854-855

17. Kim R, Emi M, Tanabe K (2007) Cancer immunoediting from immune surveillance to immune escape. Immunology 121(1):1-14

18. Lughezzani G, Burger M, Margulis V, Matin SF, Novara G, Roupret $M$ et al (2012) Prognostic factors in upper urinary tract 
urothelial carcinomas: a comprehensive review of the current literature. Eur Urol 62(1):100-114

19. Margulis V, Youssef RF, Karakiewicz PI, Lotan Y, Wood CG, Zigeuner R et al (2010) Preoperative multivariable prognostic model for prediction of nonorgan confined urothelial carcinoma of the upper urinary tract. J Urol 184(2):453-458

20. Favaretto RL, Shariat SF, Savage C, Godoy G, Chade DC, Kaag $M$ et al (2012) Combining imaging and ureteroscopy variables in a preoperative multivariable model for prediction of muscleinvasive and non-organ confined disease in patients with upper tract urothelial carcinoma. BJU Int 109(1):77-82

21. Messer JC, Terrell JD, Herman MP, Ng CK, Scherr DS, Scoll B et al (2013) Multi-institutional validation of the ability of preoperative hydronephrosis to predict advanced pathologic tumor stage in upper-tract urothelial carcinoma. Urol Oncol 31(6):904-908

22. Stein B, Schrader AJ, Wegener G, Seidel C, Kuczyk MA, Steffens S (2013) Preoperative serum C-reactive protein: a prognostic marker in patients with upper urinary tract urothelial carcinoma. BMC Cancer 13:101

23. Yates DR, Hupertan V, Colin P, Ouzzane A, Descazeaud A, Long JA et al (2012) Cancer-specific survival after radical nephroureterectomy for upper urinary tract urothelial carcinoma: proposal and multi-institutional validation of a post-operative nomogram. Br J Cancer 106(6):1083-1088

24. Jeldres C, Sun M, Lughezzani G, Isbarn H, Shariat SF, Widmer $\mathrm{H}$ et al (2010) Highly predictive survival nomogram after upper urinary tract urothelial carcinoma. Cancer 116(16):3774-3784

25 Youssef RF, Krabbe L-M, Shariat SF, Lotan Y, Sagalowsky AI, Raman J et al (2015) TALL score for predictionof oncological outcomes after radical nephroureterectomy for high-grade upper tract urothelial carcinoma. World JUrol 33(12):1965-1972

26. Roscigno M, Brausi M, Heidenreich A, Lotan Y, Margulis V, Shariat SF et al (2011) Lymphadenectomy at the time of nephroureterectomy for upper tract urothelial cancer. Eur Urol 60(4):776-783

27. Xylinas E, Rink M, Margulis V, Faison T, Comploj E, Novara G et al (2013) Prediction of true nodal status in patients with pathological lymph node negative upper tract urothelial carcinoma at radical nephroureterectomy. J Urol 189(2):468-473

28. Kates M, Sopko NA, Matsui H, Drake CG, Hahn NM, Bivalacqua TJ (2016) Immune checkpoint inhibitors: a new frontier in bladder cancer. World J Urol 34(1):49-55

29. Sung HH, Jeon HG, Jeong BC, Seo SI, Jeon SS, Choi H-Y et al (2015) Clinical significance of prognosis using the neutrophillymphocyte ratio and erythrocyte sedimentation rate in patients undergoing radical nephroureterectomy for upper urinary tract urothelial carcinoma. BJU Int 115(4):587-594

30. Kim M, Moon KC, Choi WS, Jeong CW, Kwak C, Kim HH et al (2015) Prognostic value of systemic inflammatory responses in patients with upper urinary tract urothelial carcinoma. World J Urol 33(10):1439-1457

31. Dalpiaz O, Pichler M, Mannweiler S, Martín Hernández JM, Stojakovic T, Pummer K et al (2014) Validation of the pretreatment derived neutrophil-lymphocyte ratio as a prognostic factor in a European cohort of patients with upper tract urothelial carcinoma. Br J Cancer 110(10):2531-2536 